\title{
Pragmatic Research on Context Modeling and Use
}

\section{La recherche pragmatique sur la modélisation et l'utilisation du}

\section{contexte}

\author{
Patrick Brézillon ${ }^{1}$, Roy M. Turner ${ }^{2}$ \\ ${ }^{1}$ Sorbonne Université, CNRS, LIP6, F-75005 Paris, France, Patrick.Brezillon@lip6.fr \\ ${ }^{2}$ School of Computing and Information Science, University of Maine, Orono, Maine, USA, rturner@maine.edu
}

\begin{abstract}
We argue for the centrality of a pragmatic approach to modeling and using context as a means of unifying research along all axes of context-related research: formal, cognitive, and pragmatic. After briefly discussing the evolution of the research on context in the past $25+$ years, we describe the case for a focus on pragmatic research (e.g., applications) going forward. We then give three illustrative examples of pragmatic approaches leading to implementations.

RÉSUMÉ. Nous défendons la centralité d'une approche pragmatique de la modélisation et de l'utilisation du contexte comme moyen d'unifier la recherche sur les axes de la recherche liés au contexte: formel, cognitif et pragmatique. Après avoir brièvement discuté de l'histoire et l'évolution des recherches sur le contexte ces 25 dernières années, nous décrivons les arguments pour mettre un accent sur l'approche pragmatique (par l'exemple d'applications). Nous donnons ensuite trois exemples illustratifs d'approches pragmatiques qui ont aboutis à des implémentations.

KEYWORDS. context modeling and use, pragmatic approach, artificial intelligence, contextual graphs, contextmediated behavior, context-based reasoning.

MOTS-CLÉS. modélisation et utilisation du contexte, approche pragmatique, intelligence artificielle, graphes contextuels, comportement médiatisés par le contexte, raisonnement basé sur le contexte.
\end{abstract}

\section{Introduction}

For much of its early history, there was a healthy tension in the field of Artificial Intelligence (AI) ${ }^{1}$ between formal theories (e.g., theorem proving rooted in formal logic) and system engineering and appeals to "softer" theories arising from the emerging field of cognitive science (e.g., meansends analysis). By the 1980s, however, the balance had shifted toward formal, engineered systems, with knowledge and reasoning methods largely based on theorem proving, rule-based expert systems, Bayesian networks, and fuzzy logic.

Unfortunately, formal/mathematical systems of this sort can only go so far. Their use faces severe limits in the real world, which is extraordinarily complex and messy, with missing information, uncertainty, and imprecision, and with the presence of other, possibly unknown and unpredictable, agents and processes acting to change the world in unexpected ways. It is all but impossible to create general models of the world and reasoning mechanisms that can guide an agent's or application's behavior so that it is appropriate for all the situations it may encounter.

The problem, then, is to transform formal, theoretical knowledge about operating in a domain into operational knowledge about how to perform in the particular context faced by an agent or application in a particular situation. This contextualization process adapts the agent's knowledge to

\footnotetext{
${ }^{1}$ Al refers here to the entire field, not just its subfield machine learning (which itself includes deep learning/neural networks). As AAAl (Association for the Advancement of Artificial Intelligence) states, Al is the "scientific understanding of the mechanisms underlying thought and intelligent behavior and their embodiment in machines" (from aaai.org).
} 
the task at hand, the situation, the local environment, any users' preferences, and the system's and users' past experiences. It requires attention to context as an object of study in its own right.

Context began to be treated seriously as a subject of study in the early 1990, and this has continued until the present, with work primarily centered around the interdisciplinary community associated with the CONTEXT conference series. ${ }^{2}$ However, even though it is critical to the success of real-world AI systems, there is still no universal definition of context, as pointed out by Bazire and Brézillon [1]. They found in 2005, 166 definitions of context in the literature; as of 2021, the number had grown to 267. Similarly, there is no single definition of contextual knowledge, that is, the knowledge an agent needs about a context and about how to behave while in it.

If we focus on a pragmatic view of context - how context and contextual knowledge affects and should be modeled in real-world applications and agents - then the definitions at least begin to converge. Here, we can view the agent's knowledge as its mental model of how to realize its task in the local environment, a model that is developed by accumulated experiences, i.e. contextualized knowledge or operational knowledge. This is different than the traditional view of a knowledge base or axiom set divorced from how that knowledge is used. A mental model implies a focus on knowledge's operational and dynamical organization, which is different from agent to agent and that is built from the agent's experience of performing tasks in different contexts.

The emphasis in the pragmatic view of context in science and engineering is related to the notion of practice versus procedure [3]. Generally, enterprises develop procedures to cover a class of similar problems. However, a procedure never totally matches any particular situation at hand, and so it must be adapted each time. Agents use the procedure as a framework on which to build a solution to the task given the specifics of the context: a practice. A practice captures advantages and disadvantages of the situation at hand during task realization, whereas a procedure captures only the abstracted solution across many different possible contexts. Thus, a practice is a contextualized procedure. There are therefore as many practices as contexts.

The pragmatic approach aims to solve problems in a sensible way that suits the conditions that really exist now, rather than obeying fixed theories, ideas, or rules. Pragmatic approaches aim to integrate research, policy, and practice. The philosophy of pragmatism points out the concretization of ideas by acting on them to actually check them in human experiences (see Wikipedia). Although pragmatics usually arises in linguistics and semiotics, its interest for us is that it allows studying the ways in which context contributes to meaning. Pragmatics encompasses speech act theory, conversational implicature, speech in interaction and other approaches to language behavior in philosophy, sociology, linguistics and anthropology [6]. Pragmatics studies how the transmission of meaning depends not only on structural and linguistic knowledge (e.g., grammar, lexicon, etc.) of the speaker and listener, but also on the context of the utterance, any pre-existing knowledge about those involved, and other factors. In this respect, pragmatics explains how language users are able to overcome apparent ambiguity, since meaning relies on the manner, place, time, etc. of an utterance. Our goal is to show that pragmatic approaches would have similar effects in AI, in particular, on context research.

In the rest of this paper, we first briefly discuss the evolution context research over the past 25 years. ${ }^{3}$ We then present our proposal for the future direction of context research in general and

\footnotetext{
${ }^{2}$ International and Interdisciplinary Conference on Modeling and Using Context.

${ }^{3} \mathrm{~A}$ longer, more complete version of this is forthcoming.
} 
context for AI in particular. We close with a brief look at three illustrative examples of pragmatic context research.

\section{Context research: The first $\sim 25$ years}

While natural language researchers have been concerned with context in a narrow sense for many years, context first appeared as an important challenge in AI itself at the International Joint Conference on AI in 1993 (IJCAI-93). In his seminal paper, McCarthy [5] introduced the relation $i s t(c, p)$, which states that a proposition $p$ is true in context $c$, and concluded that: a context is always relative to another context; contexts have an infinite dimension; contexts cannot be described completely; and when several contexts occur in a discussion, there is a common context above all of them into which all terms and predicates can be lifted. As a consequence, one cannot speak of context outside of its own context.

At the IJCAI-93 Workshop on Modeling and Using Context [2], intense discussions identified two different viewpoints on context. First was the formalists' viewpoint (e.g., [5]), which assumed human reasoning (and, thus, computer-based reasoning) is describable in terms of logic and formal (proof) procedures. Thus, context is useful in representing and reasoning about a restricted state space within which a problem can be solved. Second was the cognitive science-based researchers' viewpoint (e.g. [7]), for whom getting something to actually work may not be formalizable because relatively little is known about how human reasoning works, but for whom a goal was to keep the system "cognitively plausible" — both as a goal itself and as an important consideration for systems that work with humans either as assistants or co-workers or for systems that need to acquire knowledge from humans.

Context is a concern not only for AI theorists and practitioners, but also for a wide range of disciplines including psychology and cognitive science, linguistics and natural language processing, neuroscience, anthropology, sociology, organizational behavior, and philosophy. This community was fostered and made concrete by the CONTEXT series of biennial conferences, beginning in 1997 and continuing to the present. Other specialized research communities interested in context have arisen over the years (e.g., context-aware computing, ubiquitous computing, etc.), but CONTEXT remains the backbone of the interdisciplinary context community. Thus, a pragmatic view of context will interest a large community of scientists and engineers.

There has been a shift in emphasis and concerns over time. At the first conference in 1997, roughly $40 \%$ the papers had to do with communication (including NLP and most cognitive science approaches), $16 \%$ with reasoning (formal aspects), and $44 \%$ with what might be called "activity" (applications, user interfaces, etc.). By 2017, this had changed to $25 \%$ in communication, $10 \%$ in reasoning, and 65\% in "activity". This was driven in large part by the rapidly-growing importance of information and communication technology (ICT) over this time. Since ICT relies on inventions, immediate implementations, and adoption by end-users, it is not concerned with formal aspects of reasoning or cognitive plausibility.

\section{Context use and modeling: Going forward}

The tension between the three axes of context research, formal ("hard" sciences), cognitive ("soft" sciences), and applications, is as old as AI itself, reflected in the "neat" (formal) vs "scruffy" (applications, cognitive) divide in AI researchers. However, we argue that going forward, the context community focus instead on pragmatic approaches: research that is mainly concerned with creating applications and agents, but that use ideas and techniques from the formal and cognitive axes as well. Even if methods and tools developed in linguistics and other related domains turn out not to be applicable to AI, the philosophy of the pragmatic approach seems to be largely shared. 
The pragmatic approach covers a large part of the formal approach. Indeed, for one of us, our interest in modeling and using context in real-world applications came from an initial interest in the mathematical modeling of calcium metabolism as a self-oscillating nonlinear system (nonlinear differential equations studied through numerical simulation) and the encountered limits of theoretical tools when they were applied in this application. This led to exploring the possibility of coupling theoretical tools with emerging AI tools such as expert systems, although the emphasis was still on formal modeling of knowledge and of reasoning. However, it was simply not possible to deal with the AI requirements of modeling real systems such as this in a formal representation.

The pragmatic approach also covers a large part of the cognitive approach. One of us (Turner) came to research on context-based reasoning for real-world systems via a cognitive approach focused on modeling how humans store and reuse problem-solving knowledge (case-based reasoning and his own generalization of it, schema-based reasoning [9]). However, as his research evolved away from a system that interacted with humans (a medical diagnostic reasoner) to one meant to control autonomous underwater vehicles in the real world, pragmatic issues became paramount rather than strict attention to cognitive plausibility. The resulting approach was informed both by cognitive science as well as formal work (frames, formal ontologies, fuzzy logic, and more recently deep learning), but the goal was and continues to be to capitalize on the strengths of formal and cognitive research to inform and develop a pragmatic solution to actually solve real-world problems.

The pragmatic side is inherent to AI, with or without context. Much of the work in other communities tends to simplify what context is for the often rather narrow, circumscribed purpose at hand, most often having to do with simple non-AI software systems. New paradigms of context and contextual knowledge can adapt and adopt formal and cognitive context research to the needs of intelligent systems, while remaining grounded by the need to operate in the real world. Pragmatic approaches such as this (including the ones described in the next section) have often been reported in, for example, the CONTEXT conferences. Since pragmatic research is on focused solving an existing problem, the solution may be reused by other researchers with similar problems. It is a way to step back to generalize and abstract the solution based on what has been learned in solving a real problem.

The center of gravity of the interdisciplinary nature of the context community is moving regularly toward applications. We believe this has been healthy for the community and that focusing on the pragmatic approach can be a key way to overcome the tendency of the three initial approaches to become "islands" and to ensure the future health of context research across all of the different axes by a kind of diffusion process, thanks to the pragmatic approach.

\section{Examples of pragmatic context research}

In this section, we briefly describe three conceptual frameworks that progressed to the implementation level as examples of the kind of pragmatic research we are talking about. The examples we have selected attack different problems in a wide range of fields (from medicine to automated intelligent agents) in different ways. They all share some key features, including: focusing on solving a real-world problem; recognizing the usefulness of explicit context representation; and drawing from formal, cognitive, and/or applications research as needed to solve the problem. Real-world applications do not allow simplifications, approximations, and simplifying assumptions accepted when a theory fails to represent the real.

Contextual graphs [3]. A contextual graph is a directed series-parallel acyclic graph that represents the actions to undertake in accordance with the current context. Traversing the graph jointly builds and applies the practice according to the current context. The context of a practice is 
an ordered set of contextual elements that are instantiated during the practice development. A practice is a procedure that have been contextualized for the specifics of the situation at hand.

Context-based reasoning [4]. CxBR encapsulates into contexts knowledge about appropriate actions, procedures, and expectations as well as knowledge about possible new situations. By associating the potential future situations and their corresponding actions to specific situations, identification of a new situation can be simplified, since only a subset of all possible situations is applicable under the current situation. A context in CxBR contains the specifics tools and methods needed for problem solving in that context.

Context-mediated behavior [9]. CMB continually diagnoses an agent's current situation as an instance of known (from past experiences or humans) classes of situations represented as contextual schemas. Knowledge in these c-schemas then guides all facets of the agent's behavior in a contextappropriate manner: which goals to work on, how to handle unanticipated events, how to set parameters that affect behavior, and which procedural schemas (hierarchical plan-like knowledge structures) to use to achieve goals. A particular context is built using c-schemas as building blocks once the real situation has been identified.

\section{Conclusion}

The context of a task has important, inescapable consequences for how that task should be done. The context-specific nature of task realization means that general-purpose procedures need to be contextualized to become efficient practices. Developing methods for doing this requires an explicit focus on context as an object of study in its own right.

We urge a focus on a pragmatic approach to the problem based on using artificial intelligence to solve real-world problems. Having the constraint of solving a real-world problem means that pragmatic research must adapt existing approaches in addition to inventing new techniques for problem solving, both based on context modeling and use. We believe that focusing more attention on pragmatic approaches will reward the context community as it goes forward. By naturally concentrating on different problems, pragmatic approaches already have developed different formalisms (e.g., from CMB, $\mathrm{CxG}, \mathrm{CxBR}$ ), but all anchored to the real world. This facilitates identification of similarities and differences in the models, which can point out both where complementary parts of models can be transferred between approaches, as well as where there are incommensurate parts indicate, which indicate where additional research should be focused or where the models can be combined in a multi-faceted way, conditionalized for problem type. Pragmatic approaches also naturally foster collaboration as researchers encounter similar challenges and problems. Since the space of problems addressed even within the three formalisms mentioned above include autonomous agents, agents that collaborate with each other (multiagent systems), and systems that collaborate with humans, pragmatic research opens the way for a coherent model of context for systems spanning the autonomous - collaborative spectrum.

Consequently, we propose a new identity for our interdisciplinary context community as we go forward:

An interdisciplinary forum for exchanging practical experience modeling and managing context in real-world systems.

\section{Bibliography}

[1] Bazire, M., Brézillon, P.: Understanding context before to use it. In: Dey, B. Kokinov, D. Leake, R. Turner (eds.) Modeling and Using Context (CONTEXT-05), LNCS, vol. 3554, pp. 29-40. Springer Verlag, Heidelberg (2005). 
[2] Brézillon, P. (Ed.): Proceedings of the IJCAI-93 Workshop on Using Knowledge in its Context. Rapport de Recherche $N^{\circ}$ 93/13, LAFORIA, Université Paris VI, Paris, France (1993).

[3] Brézillon, P.: Context modeling: Task model and model of practices. In: Kokinov et al. (Eds.) Modeling and Using Context (CONTEXT-07), LNAI, vol. 4635, pp. 122-135. Springer Verlag (2007).

[4] Gonzalez, A J and Ahlers, R (1998). Context-based representation of intelligent behavior in training simulations. Transactions of the Society of Computer Simulation 15 (4) pp 153-166.

[5] McCarthy, J.: Notes on formalizing context. Proceedings of the 13th IJCAI, vol. 1, pp. 555-560 (1993).

[6] Mey, J.L. (1993) Pragmatics: An Introduction. Oxford: Blackwell (2nd ed. 2001).

[7] Newell, A., Simon, H.A.: Computer science as empirical inquiry: Symbols and search. Communications of the $A C M, 19$ (3), pp. 113-126 (1976).

[8] Turner, R.M.: Adaptive Reasoning for Real-World Problems: A Schema-Based Approach, R.M. Turner. Lawrence Erlbaum Associates, Inc., Hillsdale, NJ, 1994.

[9] Turner, R.M.: Context-mediated behavior. Modeling and Using Context, vol. 1-1, March, 2017. 